\title{
The principle and method of urban ecological restoration planning
}

\author{
Xia Xiaotang \\ ${ }^{1}$ School of Urban Design, Wuhan University, 8 South Donghu Road, Wuhan 430072, China \\ ${ }^{2}$ College of urban construction, Wuhan University of Science and Technology, Huangjia Lake Road, \\ Wuhan 430000, China \\ xiaxiaotang2010@qq.com
}

Keywords: ecological restoration; landscape planning; ecological environment deterioration

Abstract. Faced with the deteriorating ecological environment, people began to apply the principles of ecology to urban landscape planning and construction, which make a number of methods of ecological planning and design. Ecological restoration design is one of them. This paper indicates the background and connotation of urban ecological restoration planning, also mentions several cases to illustrate methods in the three different levels.

As you can see from the Google maps, along both sides of the river are beaches, ponds, or salt field in 1995. Until 2002, there are a certain buildings, but constructions have been basically full coverage by 2014. Because of many years of rapid economic development and the process of urbanization has brought a lot of urban diseases. Urban diseases come from all aspects, of which the construction of urban environment and urban landscape are obvious important aspects. At the same time, the deposition of the river is also very prominent. As is well known, the river is the most common and complex problem in the ecological environment. Good ecological environment is the fairest public product, is the most concerned problem of people's livelihood ${ }^{[1]}$.

《The national planning of urban ecological protection and construction (2015-2020) 》analyzes the general situation of our country's construction in urban ecological environment. Planning Outlines the overall situation of urban ecological system at the present stage: Overall trend of urban ecological environment deterioration has not been curbed, urban spatial spread disorderly, total ecological space is insufficient, some unreasonable city layout, serious situation of environmental pollution, smog, heat island effect, water pollution, soil pollution, lag of urban infrastructure construction, low efficiency of resources, energy, huge challenges of urban ecological security faced by urban agglomeration and mega-city. Also it pointed out that, at present, the most prominent problem is as follows: imbalance of the city and regional ecological relationship, serious occupation of urban space; degradation of urban ecological function, decrease of biodiversity; total lack of urban garden and Greenland, requirement of improving the service ability; low efficiency of resources energy, overload volume of urban ecological environment, grim situation of environment pollution in cities; insufficient ability of city ecological protection and construction, and unsound mechanism of the management system.

The connotation of ecological restoration

In this case, we should do? The ecological restoration is a commonly used gimmick. From the aspect of connotation, ecological restoration is a kind of intervention which is under the way of 
ecological restoration. Mainstream understanding of ecological restoration is mainly use the resilience of the ecosystem, supplemented by artificial measures. Which make the destruction of ecological environment gradually recover, or make the ecological system develop in the direction of good circulation. At present, urban ecological restoration relies primarily on the ability of ecological system self-organization and self-adjusting, artificial regulation and intervention is complementary to the outside world ${ }^{[2]}$. Regulation require multidisciplinary collaborative of the outside world, including ecology, soil and water conservation science and landscape architecture is the most basic subject, associated with a dozen of other subjects related to ecological restoration, and intervention through different angles.

The theory of urban ecological restoration is originated in the United States in 1930, which has a bigger development in the 80 's. It should be said that ecological restoration is the product of a post-industrial environment change ${ }^{[3]}$. The large-scale industrialization brings a series of environmental problems, in such a concept. In fact our country also faces such period at present. After rapid urban development and economic development, China has entered the phase of an urgent need of ecological restoration. China is one of the most serious countries that have ecological environment problems. The types of ecosystem degradation are various. Meanwhile, restoration ecology research and implementation work are carried out very early. We can recall that work related to urban ecological restoration can be traced back to the 1950's. The 50's to 80's ecological restoration is mainly focused on the acquisition of general information, and repair of degraded ecosystem to ascertain small scale, such as the typical hill forestation and vegetation restoration. In the 70's, there are forest construction in three north shelter, to vigorously develop ecological restoration in the 80's. In the process of our country's rapid economic growth, ecological degradation and environmental pollution are very serious, especially forest, coastal zone, grassland, desert, farmland which have substantial degradation phenomenon ${ }^{[4]}$. This situation puts forward how to build ecological restoration theory direction and methods of the system with the situation of China's. According to the height of the construction of ecological civilization, a complete system of ecological civilization needs to be built, to protect the ecological environment of such a higher level. At the same time, it can also see that the ecological restoration is a gradual process.

Urban ecological restoration basically relies on self-repair ability of ecological system itself, supplementing by the appropriate artificial adjustment from the outside. It can recover the original ecological system in order to conserve soil and water, adjust microclimate, purifying environment, and maintain the ecological function of biodiversity. Ecosystem restoration aims to repair and improve the function of ecological system continuously, not fully to recover the ecological system to its original state. Our ecological restoration is definitely not like the restoration of cultural relics, which pursue "the more vivid the better". In fact our target is to restore the function and structure of ecological system, and its ability to repair by itself - that is ecological function.

The main contents of National ecological protection and construction planning carry out the protection and construction with natural ecological resources as the object. The project has clarified about the object of the ecological protection and ecological restoration, and defined urban natural ecological resources, including green land, soil, air, water, plants and animals, and the landform conditions and so on. These are all attentions of ecological rehabilitation, and also the basic conditions for existence and development of urban compound ecosystem. All these determine the spatial pattern and form of urban development.

So what is the urban ecological restoration planning? The urban ecological restoration planning is to solve the most prominent problem for urban ecological field. It also is the problem oriented 
primarily. The plan usually speaks two directions, one is problem oriented, and the other is goal oriented. While, ecological restoration of goal-directed is hard to define, however, if the target is in the original natural environment, in fact is hard to achieve. As an intermediate link, urban ecological restoration may simply be a phased goal, but not the ultimate goal. So, in my personal view, ecological restoration planning should also be predominantly problem oriented.

The main purpose of urban ecological restoration is to repair and reconstruct the damage natural ecosystem. The main target of planning are urban natural ecological resources, urban ecological space, and ensuring the safety of urban ecological system for the green infrastructure. Besides, it is in order to improve the urban ecological function, improve the ecological system of self-organization self-adjusting and self-healing ability, promote the healthy development of ecological system, optimize the urban ecological space-structure, and improve the human living ecological environment.

Therefore, firstly ecological restoration is to perfect the urban ecological function, improve the self-organization, self-control and self-repair ability of ecological system. We do provide the conditions for ecosystem's recovery ability. We make its have immunity, then ecological system can achieve a virtuous circle. At the same time, we also need to optimize the urban ecological space. And the ultimate goal of this work is to improve the ecological environment for human settlement. Ecological environment for human settlement includes three subjects (architectures, gardens, urban planning), which is created by Mr. Wu Liangyong.

Three levels of urban ecological restoration planning

The regional level

Regional level can be a planning zone or a larger area, according to the actual situation. The key problems of this level are to clarify the natural and culture sensitive area, and to determinate of reasonable urban growth boundary. For example, the case is Gui' an New Area on the west side of GuiZhou. The master plan of Gui' an new area needs to determine $t$ of urban construction area. The work is to do related research before determine the overall urban construction land, including draw boundary lines of water reserves in the area firstly, mountain conservation area, vegetation protection area, national scenic area, national forest park, etc.

In addition, we also analyses the water system, which needs to include the water ecological system into green space system, coupling with urban development and construction. Then, overlay the resources in most protection-value in this area and form the banning construction area. Which do provide supports for further clarify city constructed areas, find space for urban development scientifically and reasonably. In this way, we can join the city and natural mountain environment well.

Another case is Manas located in Xinjiang. We cooperate two plans (one is the master plan of Manas county, the other is a Manas city's wetland park plan). Through Manas city's wetland park plan, all the river and the surrounding region can be protected. After these, it has played a very good supporting effect to determine the boundary of the city development area.

The city level

This level mainly aims at constructed area in urban planning, coordinating with artificial environment and natural environment, and reducing the negative effects of urban development on natural circulation process, as soon as possible to restore the ecological system as the main target. Specific methods are as follows: 
I To do analysis of natural ecological resources, especially the water ecological elements, to delimit the ecological control line.

I To restore the damaged ecological system,

I To build urban green infrastructure network, in order to adapt to the ecological space of natural process,

I To optimize the pattern of urban green space system.

Here is the case, located in Haidian district in northern mountainous area of urban green heart in Beijing. Recorded in history, Beijing Haidian district is full of wetlands, and $50 \%$ of the land is water, but now more than $90 \%$ of the land is not water. The plan is mainly to analyze and compare important ecological factors of the rivers, drainage, river canal, wetlands, rice paddies, and finally determine nearly 60 square kilometers into the development of the green heart. At the same time, Cuihu wetland is analyzed in local areas.

A series of research has been done to find out how to use the wetland park to recover the drainage of wetlands in this area. After the study of history, the research analyzes the original historical environment, then put forward the target to restore its primitive natural ecological environment basically (here mainly refers to the area and proportion of how to recover ecological elements. But to recover its internal ecological structure is difficult to achieve). In addition, we also recover the original Jingxi rice from the aspects of culture, and renew the original villages. Through the whole series of work, finally destructed natural factors have corresponding recovery, and the water quality is purified. The environment in whole area is very good; a lot of people are spontaneously to play there. At the same time, also it left a very good case for the future development of Zhongguan Village in Beijing.

Another case is ecological garden city construction plan in Anshan city. Anshan is the steel capital of the republic. In the process of nearly one hundred years of construction, Anshan has left quite a lot of rubbish, mine, and mine. In 1981, the local natural mountain is saved very well, also in 1996 has a little invasion, but it has been completely invasion in 2012.

Under such circumstances, we analyze the security pattern of biodiversity, regional industrial pollution, mountain landscape protection, and heat island effect, which is the basis for the rest of the plan. Through this process of analysis, how to solve the problem of Anshan iron and steel plant and the peripheral, problem of green wedge to protect ecology and ventilation corridor, and the problem of heat island effect are gradually put forward.

Ground level

The application of putting landscape design idea into sponge city in the ground level can be found from in all aspects, such as the square, green space areas and sidewalks, roads, roof. In the city level, in the process of urban internal ground design, the basic idea is combining landscape green space and the concept of sponge city, then take water ecological infrastructure technology to construct the specific system of sponge city, and maintain hydrological signs and natural characteristics before the development. This is our cases in Sanya. We control various aspects of the road, building, district, city water system and so on. Combining philosophy and concept of planning has been implemented of the way.

\section{Conclusion}

In the end, I think the main guidance of ecological restoration planning is to emphasize the outstanding problems in urban ecological field. Restoration and reconstruction of damaged natural ecosystem are the main purposes. To improve the urban ecological function, to improve the 
self-organizing, self-control and self-repair ability of ecology, to promote healthy development of the ecological system, to optimize the structure of urban ecological space, and to improve the ecological environment for human habitant are the basic goals.

\section{References}

[1] Chen Bo, Bao Yizhi, The Application of Ecological Restoration Design in Urban Landscape Planning, Chinese Landscape Architecture 2003, 19(7)

[2] Jiang Xiujuan, Zheng Bohong, Pan Qianhua, The study of restoring city's abandoned area ecological planning abroad, Chinese and Overseas Architecture, 2007(2), pp.6-8

[3] Wu Xiuying, Zhao Xinggang, The application of restoration of ecological theory in city planning, Shanxi Architecture, 2009.5, Vol 35, No.14, pp 5

[4] Wang Linlin, Hu Zhenqi, Ecological restoration plan for resource-exhausted mining areas and case studies, Modern Urban Research, 2009(7) 